\section{Comments on an Article by Kamalian et al}

We read with great interest the article by Kamalian et al. ${ }^{1}$ The authors found, in a carefully selected patient group, that CT perfusion mean transit time maps optimally distinguished benign oligemia from true "at-risk" ischemic penumbra. They postprocessed the same dynamic CT data with 2 commercial software packages from the same vendor, containing a standard and a delay-corrected (DC) deconvolution algorithm, respectively.

For each algorithm considered separately, receiver operating characteristic (ROC) analysis yielded a significantly higher area under the curve for absolute and relative MTT than for the other CTP parameters for identification of brain tissue destined to infarct (all $P$ values $<.01$ ). The authors also found that thresholds needed to be adapted. They reported that "absolute and relative MTT thresholds for defining penumbra were 12 seconds and $249 \%$ for the standard and 13.5 seconds and $150 \%$ for the delay-corrected algorithms, respectively." That thresholds may vary considerably among different approaches and implementations is well known, but their results appear to have an internal discrepancy that requires clarification. Relative MTTs were calculated by normalizing the ischemic MTT to the one for the corresponding anatomy on the contralateral side. Therefore, one can reversely deduce that the average MTT of the normal brain by using the standard algorithm in their data was approximately 4.8 seconds ( $12 / 2.49$ seconds); the one in the delay-corrected version, however, would have to be approximately 9 seconds (13.5/1.5 seconds). Nine seconds would be in total disagreement with basically all normal MTT values that can be found in the literature (eg, Wintermark et $\mathrm{al}^{2}$ ), which are more in the range of $4-6$ seconds (in agreement with the results of the standard algorithm).

In addition, a DC algorithm would tend to have an even shorter MTT. ${ }^{3}$ The gross whole-brain transit time can be estimated from the peak time difference of the arterial input function and the venous outflow function. In our experience, this difference is typically between 6 and 8 seconds; tissue MTT must be shorter. Figures 3 and 4 in a recent review article coauthored by 2 of the authors of the present study clearly confirm this. ${ }^{4}$ If there was no accidental misreporting of numbers, this discrepancy definitely requires an explanation and discussion.

Furthermore, the authors argue at length that DC algorithms are superior to standard ones because they are better adapted to the variable arrival times. We fully agree. Their data, however, appear to demonstrate the opposite. All areas under the curve and specificities for the standard algorithm are consistently higher than those for DC. If the next accurate parameter was $\mathrm{CBF}$, as they report, and the difference between 0.76/0.78 (MTT standard) and 0.73/0.74 (CBF standard) was significant at $P<.01$, then clearly the difference between 0.76/0.78 (MTT standard) and 0.72/0.71 (MTT DC) will have similar or higher significance. If one were to use the authors' reasoning about optimal performance, one could also draw the conclusion that the CBF of standard deconvolution software (CTP3 "Std," GE Healthcare) performs better than the MTT of delay-corrected software (CTP5 "DC"; GE Healthcare). We believe this issue requires further explanation and discussion.

\section{References}

1. Kamalian S, Kamalian S, Konstas AA, et al. CT perfusion mean transit time maps optimally distinguish benign oligemia from true "at-risk" ischemic penumbra, but thresholds vary by postprocessing technique. AJNR Am J Neuroradiol 2012;33:545-49

2. Wintermark M, Flanders AE, Velthuis B, et al. Perfusion-CT assessment of infarct core and penumbra: receiver operating characteristic curve analysis in 130 patients suspected of acute hemispheric stroke. Stroke 2006;37:979-85

3. Abels B, Klotz E, Tomandl BF, et al. Perfusion CT in acute ischemic stroke: a qualitative and quantitative comparison of deconvolution and maximum slope approach. AJNR Am J Neuroradiol 2010;31:1690-98

4. Konstas AA, Wintermark M, Lev MH. CT perfusion imaging in acute stroke. Neuroimaging Clin N Am 2011;21:215-38, ix

P. Schramm
Department of Neuroradiology
University Medicine Goettingen
Goettingen, Germany
E. Klotz
Siemens Healthcare Sector
Forchheim, Germany

http://dx.doi.org/10.3174/ajnr.A3153 